\title{
Resveratrol attenuates the inflammatory reaction induced by ischemia/reperfusion in the rat heart
}

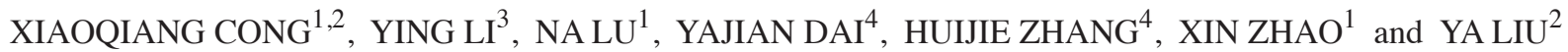 \\ ${ }^{1}$ Department of Cardiology, Bethune First Hospital of Jilin University; ${ }^{2}$ Department of Nutrition and Toxicology, \\ School of Public Health, Jilin University, Changchun, Jilin 130021; ${ }^{3}$ Department of Emergency Internal Medicine, \\ The People's Hospital of Jilin Province, Changchun, Jilin 130021; ${ }^{4}$ Department of Internal Medicine, \\ The Friendship Hospital of Linjiang, Linjiang, Jilin 134600, P.R. China
}

Received July 24, 2013; Accepted February 26, 2014

DOI: $10.3892 / \mathrm{mmr} .2014 .2090$

\begin{abstract}
The role of resveratrol (Res) in inflammation induced by ischemia/reperfusion is not well understood. The aim of the present study was to investigate whether Res modulates neutrophil accumulation and tumor necrosis factor- $\alpha$ (TNF- $\alpha)$ induction in an ischemia/reperfusion-injured rat heart model. The rats were randomly exposed to sham surgery, myocardial ischemia/reperfusion (MI/R) alone, $\mathrm{MI} / \mathrm{R}+\mathrm{Res}, \mathrm{MI} / \mathrm{R}+\mathrm{Res}+\mathrm{L}-\mathrm{NG}$-nitroarginine methyl ester (L-NAME) and MI/R + Res + methylene blue (MB). The results demonstrated that compared with $\mathrm{MI} / \mathrm{R}$, Res reduced the myocardial infarct area, myocardial myeloperoxidase levels, serum creatinine kinase and lactate dehydrogenase levels, and serum and myocardial TNF- $\alpha$ production. All the effects of Res demonstrated were inhibited by L-NAME (a nitric oxide (NO) synthase inhibitor) and MB [a cyclic guanosine monophosphate (cGMP) inhibitor]. Thus, Res produces cardioprotective and anti-inflammatory effects. These effects may be associated with an increase in NO production, the inhibition of neutrophil accumulation, TNF- $\alpha$ induction and cGMP signaling pathways in myocardium subjected to MI/R.
\end{abstract}

\section{Introduction}

The inflammatory reaction induced by ischemia/reperfusion is one of the most significant factors of myocardial ischemia/reperfusion (MI/R) injury (1). In the process of

Correspondence to: Dr Xin Zhao, Department of Cardiology, Bethune First Hospital of Jilin University, 71 Xinmin Road, Changchun, Jilin 130021,

E-mail: xin_zhao2013@163.com

Dr Ya Liu, Department of Nutrition and Toxicology, School of Public Health, Jilin University, 1163 Xinmin Road, Changchun, Jilin 130021, P.R. China

E-mail: liu_ya2013@163.com

Key words: resveratrol, ischemia/reperfusion injury, neutrophil, tumor necrosis factor- $\alpha$ inflammation, various cytokines are released, including tumor necrosis factor- $\alpha$ (TNF- $\alpha$ ), interleukin (IL)-6 and IL-8 (2). TNF- $\alpha$ can trigger the inflammatory reaction caused by MI/R. In addition, vascular endothelial cell injury, and inflammatory cells, including neutrophils, activated by cytokines and adhesion molecules are also included in inflammation. Thus, TNF- $\alpha$ activity and the quantity of neutrophil infiltration can be considered as indicators of the inflammatory reaction.

Resveratrol (Res) is a polyphenolic compound, which mainly exists in red grapes and wine. In a previous epidemiological study (3) concerning the correlation between dietary habits and coronary heart disease, it was observed that amongst all the developed countries, the French consume the largest quantity of wine on average, but have the lowest morbidity rate from coronary heart disease. This phenomenon is termed the 'French paradox', which is likely to partly result from Res in wine. Res has extensive pharmacological effects, it has been demonstrated to have anticancer properties (4-7), improve ifosfamide-induced Fanconi syndrome in rats (8), treat diabetic nephropathy (9) and protect neurons $(10,11)$. A previous study has indicated that Res also exhibits anti-inflammatory effects; however, its role in mediating inflammation is not well understood (12). Therefore, the present study aimed to investigate the effect of Res on neutrophil infiltration and $\mathrm{TNF}-\alpha$ production in a rat model of $\mathrm{MI} / \mathrm{R}$, and its underling mechanisms.

\section{Materials and methods}

Reagents. Res was purchased from Sigma Chemical Co. (St. Louis, MO, USA). Myeloperoxidase (MPO) assay, creatinine kinase (CK) test and lactate dehydrogenase (LDH) assay kits were purchased from JianCheng Bioengineering Institute (Nanjing, China). A TNF- $\alpha$ ELISA kit was purchased from R\&D Systems (Minneapolis, MN, USA). L-NG-nitroarginine methyl ester (L-NAME) and methylene blue (MB) were purchased from Sigma Chemical Co. The bicinchoninic acid (BCA) protein quantification kit was purchased from Bio-Rad (Hercules, CA, USA).

Animals. A total of 50 adult male Sprague-Dawley rats (250-300 g) were purchased from the Center of Experimental 
Animals, Jilin University (Changchun, Jilin, China). All the animals used in the present study were cared for in accordance with the Guidelines for the Care and Use of Laboratory Animals published by the United States National Institute of Health (NIH publication no. 85-23, revised 1996), and all procedures were approved by the Committee of Experimental Animals of Jilin University.

$M I / R$ model and experimental protocol. Male Sprague-Dawley rats (250-300 g) were anesthetized intraperitoneally with $40 \mathrm{mg}$ / $\mathrm{kg}$ sodium pentobarbital (Sigma Chemical Co.). Myocardial ischemia was produced by exteriorizing the heart with a left thoracic incision followed by a slipknot (5-0 silk) around the left anterior descending (LAD) coronary artery. Subsequent to $30 \mathrm{~min}$ of ischemia, the slipknot was released and the animal received 120 min of reperfusion.

The rats were randomly assigned to five experimental groups with 10 rats per group: i) Sham group, the LAD coronary artery was exposed and a suture was passed beneath it but was not subjected to ligation and reperfusion; ii) MI/R group, LAD was ligated for $30 \mathrm{~min}$ and then allowed $120 \mathrm{~min}$ reperfusion with administration of vehicle $[0.9 \% \mathrm{NaCl}$ intravenously (i.v.)]; iii) $\mathrm{MI} / \mathrm{R}+\mathrm{Res}$ group, Res $(100 \mu \mathrm{mol} / \mathrm{l}$; i.v.) was administered 5 min prior to reperfusion; iv) MI/R + Res + L-NAME group, L-NAME (1 mmol/l; i.v.), a nitric oxide synthase (NOS) inhibitor, was administered $20 \mathrm{~min}$ prior to reperfusion. At $15 \mathrm{mins}$ after the administration of L-NAME, Res (100 $\mu \mathrm{mol} / \mathrm{l}$; i.v.) was administered. v) MI/R + Res + MB group, MB (50 $\mu \mathrm{mol} / \mathrm{l}$; i.v.), a cGMP inhibitor, was administered $20 \mathrm{~min}$ prior to reperfusion. At 15 mins after the administration of MB, Res $(100 \mu \mathrm{mol} / \mathrm{l}$; i.v. $)$ was administered.

Assay of myocardial infarct area. Following reperfusion, the myocardial infarct size was determined by means of a double-staining technique and a digital imaging system (Adobe Systems Incorporated, San Jose, CA, USA; infarct area / area at risk x 100) (13). Following reperfusion, the coronary blood flow was again blocked and Evans blue dye ( $2 \%, 4 \mathrm{ml}$ ) was injected by the rapid distribution of the right ventricle into the body. The heart was quickly removed to a $-20^{\circ} \mathrm{C}$ refrigerator for cryopreservation. The heart was cut into 1-mm slices, placed in 1\% 2,3,5-triphenyltetrazolium chloride (TTC) solution, incubated for $15 \mathrm{~min}$ and then placed in $4 \%$ formaldehyde solution (Prolabo, Fontenay-sous-Bois, France) overnight. Evans blue-stained (blue staining, non-ischemic area), TTC-stained (red staining, ischemic area) and non-TTC-stained areas (white, infarct area) were analyzed with a digital imaging system by computer. The myocardial infarct area [infarct area / area at risk x 100, (INF/AAR\%)] was calculated.

Determination of MPO levels. Following reperfusion, the myocardial tissue was placed at $-70^{\circ} \mathrm{C}$ for preservation. The MPO test kit was used to detect the level of MPO in the myocardial tissue according to the manufacturer's instructions.

Detection of $C K$ activity. Following reperfusion, blood was obtained from the carotid artery and was maintained at room temperature for $30 \mathrm{~min}$. Next, the serum was collected by centrifugation at $3,000 \times \mathrm{g}$ for $20 \mathrm{~min}$ at $4^{\circ} \mathrm{C}$ and placed

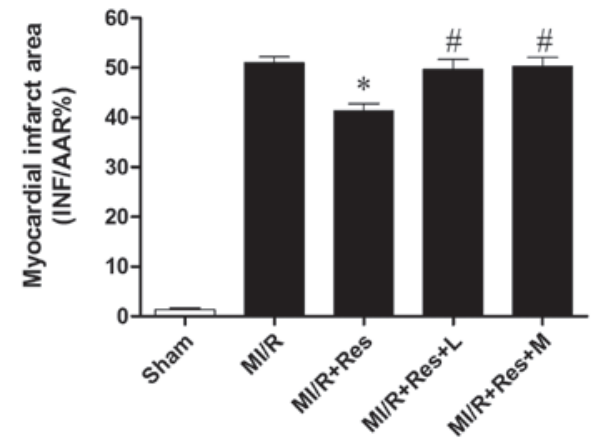

Figure 1. Comparison of INF/AAR\% in each group. 2,3,5-triphenyltetrazolium chloride-Evans blue double staining indicated that compared with the MI/R group, Res significantly reduced the infarct area, while L and M eliminated the effect of Res. ${ }^{*} \mathrm{P}<0.05$ vs. the $\mathrm{MI} / \mathrm{R}$ group and ${ }^{\#} \mathrm{P}<0.05$ vs. the $\mathrm{MI} / \mathrm{R}+$ Res group. INF/AAR\%, myocardial infarct area/area at risk; MI/R, myocardial ischemia/reperfusion; Res, resveratrol; L, L-NAME; M, methylene blue; L, L-NG-nitroarginine methyl ester.

at $-70^{\circ} \mathrm{C}$ for preservation. According to the manufacturer's instructions, the CK test kit was utilized to detect the serum CK activity.

Determination of $\mathrm{LDH}$ levels. Following reperfusion, blood was obtained from the carotid artery and was placed at room temperature for $30 \mathrm{~min}$. Next, the serum was collected by centrifugation at $3,000 \times \mathrm{g}$ for $20 \mathrm{~min}$ at $4^{\circ} \mathrm{C}$ and placed at $-70^{\circ} \mathrm{C}$ for preservation. The extent of cell injury was monitored by measuring the LDH leakage. According to the manufacturer's instructions, the CK test kit was utilized to detect the serum LDH levels.

Detection of TNF- $\alpha$ levels. Following reperfusion, the levels of TNF- $\alpha$ in myocardial tissue homogenate and serum were detected in accordance with the manufacturer's instructions. The BCA kit was used to quantify the amount of protein.

Statistical analysis. The data is presented as the mean \pm standard deviation. The significance of differences among groups was evaluated by Student's t-test for unpaired data or Dunnett's t-test for multiple comparisons preceded by one-way analysis of variance. $\mathrm{P}<0.05$ was considered to indicate a statistically significant difference.

\section{Results}

Res reduces the myocardial infarction area induced by $M I / R$. $\mathrm{MI} / \mathrm{R}$ induced a significant infarction area. Compared with the MI/R group, Res significantly reduced the myocardial infarction area. The effect of Res was inhibited by L-NAME, a NOS inhibitor. In addition, the effect of Res was evidently attenuated by MB, a cGMP inhibitor (Fig. 1).

Res inhibits neutrophil infiltration in MI/R tissue. Neutrophils contain a certain quantity of MPO, accounting for $5 \%$ of the dry cell weight. Thus, the activity of MPO in the myocardium can be considered as an indication of neutrophil infiltration. As shown in Fig. 2, the MPO activity in the sham group was relatively lower, while the MPO activity in the MI/R group was significantly increased. Res significantly decreased the 


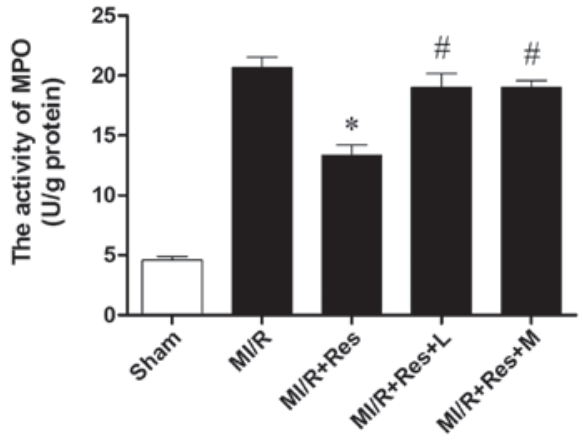

Figure 2. Comparison of MPO activity in each group. Compared with (MI/R) group, the MPO activity in the MI/R + Res group was significantly reduced. $\mathrm{L}$ and $\mathrm{M}$ eliminated the effect of Res. ${ }^{\text {}} \mathrm{P}<0.05$ vs. the MI/R group; ${ }^{*} \mathrm{P}<0.05$ vs. the MI/R + Res group. MPO, myeloperoxidase; MI/R, myocardial ischemia/reperfusion; Res, resveratrol, L, L-NG-nitroarginine methyl ester; M, methylene blue.

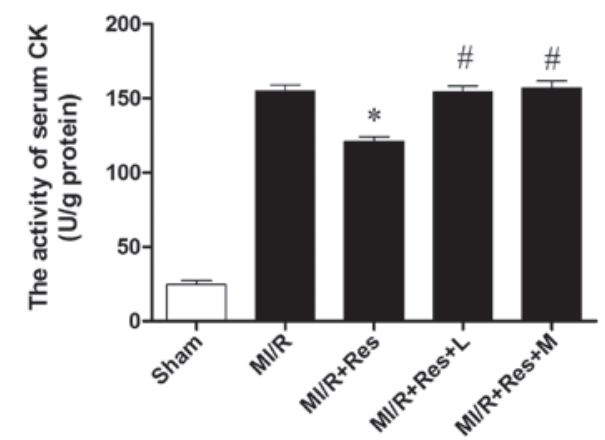

Figure 3. Comparison of serum CK activity in each group. Compared with the MI/R group, Res significantly reduced the serum CK activity. However, this effect was eliminated by $\mathrm{L}$ and $\mathrm{M}$. ${ }^{*} \mathrm{P}<0.05$ vs. the $\mathrm{MI} / \mathrm{R}$ group and ${ }^{\#} \mathrm{P}<0.05$ vs. the MI/R + Res group. CK, creatinine kinase; MI/R, myocardial ischemia/reperfusion; Res, resveratrol; M, methylene blue; L, L-NG-nitroarginine methyl ester.

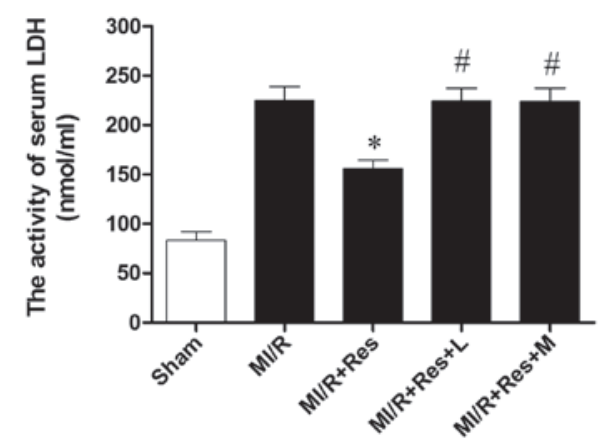

Figure 4. Comparison of serum LDH activity in each group. Compared with the $\mathrm{MI} / \mathrm{R}$ group, resveratrol (Res) significantly reduced the serum LDH activity. However, this effect was eliminated by $\mathrm{L}$ and $\mathrm{MB}$. ${ }^{*} \mathrm{P}<0.05$ vs. the $\mathrm{MI} / \mathrm{R}$ group and ${ }^{\#} \mathrm{P}<0.05$ vs. the MI/R + Res group. $\mathrm{LDH}$, lactate dehydrogenase; MI/R, myocardial ischemia/reperfusion; Res, resveratrol; L, L-NG-nitroarginine methyl ester; M, methylene blue.

myocardial MPO activity, while L-NAME and MB attenuated the effect of Res.

Res reduces the activity of serum $C K$ in $M I / R$ rats. As shown in Fig. 3, the activity of CK increased significantly in the
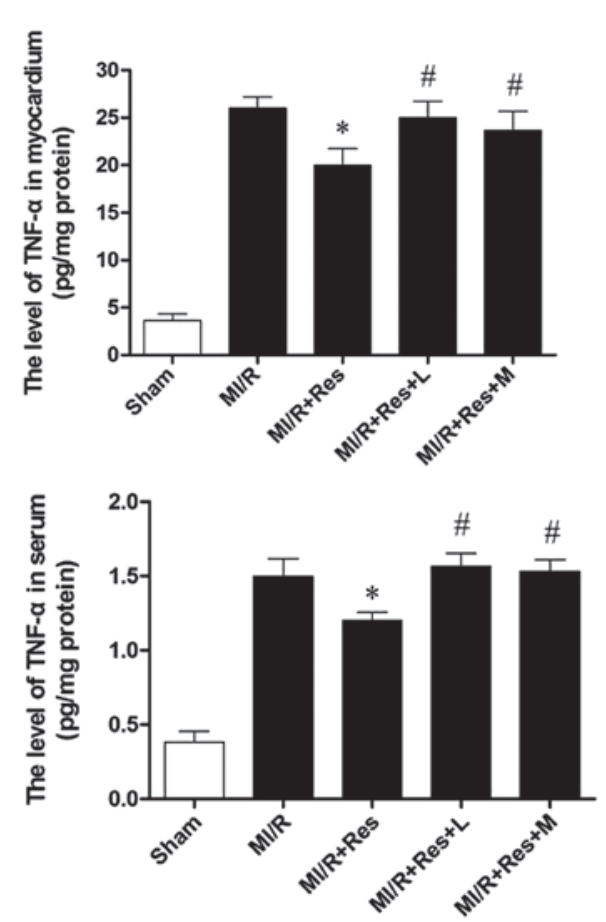

Figure 5. Comparison of levels of TNF- $\alpha$ in the myocardium and serum in each group. Compared with the MI/R group, Res reduced the TNF- $\alpha$ level significantly. $\mathrm{L}$ and $\mathrm{M}$ eliminated the effect of Res. ${ }^{*} \mathrm{P}<0.05$ vs. the MI/R group and ${ }^{\#} \mathrm{P}<0.05$ vs. the $\mathrm{MI} / \mathrm{R}+\mathrm{Res}$ group. TNF- $\alpha$, tumor necrosis factor- $\alpha$; MI/R, myocardial ischemia/reperfusion; Res, resveratrol, $\mathrm{L}$, L-NG-nitroarginine methyl ester; M, methylene blue.

MI/R group compared with the sham group. CK activity decreased significantly in the MI/R + Res group compared with the MI/R group. The effect of Res was inhibited by L-NAME and MB.

Res reduces $L D H$ levels in MI/R rats. As shown in Fig. 4, the activity of LDH increased significantly in the MI/R compared with the sham group. LDH activity decreased significantly in the MI/R + Res group compared with the MI/R group. The effect of Res was inhibited by L-NAME and MB.

Res reduces TNF- $\alpha$ levels in serum and MI/R tissue. The MI/R injury results in production of a large quantity of TNF- $\alpha$. Thus, myocardial and serum TNF- $\alpha$ levels were examined. As shown in Fig. 5, compared with the MI/R group, Res significantly decreased the levels of TNF- $\alpha$ in the myocardium and serum, which was eliminated by L-NAME and MB.

\section{Discussion}

The major findings in the present study were that Res attenuates the inflammatory reaction induced by $I / R$ injury by inhibiting neutrophil infiltration and TNF- $\alpha$ production. In addition, NO and cGMP may be important in the protective effect of Res.

The inflammatory reaction is significant in myocardial ischemia/reperfusion injury (1). The release of inflammatory cytokines and the aggregation and infiltration of inflammatory cells are the key steps in inflammation (14). 
TNF- $\alpha$ is secreted mainly by macrophages, which is likely to promote an inflammatory cascade by increasing the release of other proinflammatory cytokines and influencing neutrophil recruitment (15). TNF- $\alpha$, a significant cytokine in inflammation, is important in the initiation of the inflammation induced by MI/R (16). TNF- $\alpha$ can induce the release of other inflammatory mediators, increase the expression of cell adhesion factor and promote neutrophil adhesion to endothelial cells. In addition, TNF- $\alpha$ has a negative inotropic effect, which can inhibit myocardial contractility and lower blood pressure. TNF- $\alpha$ can also induce cardiomyocyte apoptosis and participate in ventricular remodeling (17). Previous studies indicate that the level of TNF- $\alpha$ increases significantly following MI/R (18), while the administration of TNF- $\alpha$ monoclonal antibody attenuated edema, and is conducive to cardiac function recovery (19).

$\mathrm{MI} / \mathrm{R}$ injury appears to be induced in part by neutrophil activation. The underlying mechanisms include: i) Cell damage caused by the release of oxygen free radicals, proteolytic enzymes and cytotoxic substances; ii) the released inflammatory mediators cause vascular endothelial cell damage, increased vascular permeability and edema; iii) further activation of inflammatory cells increase the inflammatory response (20); and iv) neutrophil adhesion to vascular endothelium and small blood vessel occlusion results in the no-reflow phenomenon (21).

Previous studies have demonstrated the link between neutrophil and I/R injury. Removal of neutrophils or drug inhibition of neutrophil activity has been shown to reduce I/R injury $(22,23)$. In the present study, it was identified that neutrophil accumulation and TNF- $\alpha$ production in the MI/R group increased significantly. In addition, Res reduced these effects, indicating that Res attenuated neutrophil-mediated I/R injury.

It is hypothesized that NO is closely correlated with MI/R-induced inflammation (24). Endothelial-derived NO can inhibit cell adhesion factors, including P-selectin and intercellular adhesion molecule 1 levels, thereby inhibiting leukocyte adhesion and inward membrane migration (25). Endothelial-derived NO can also inhibit the expression of TNF- $\alpha$ and other pro-inflammatory factors. Endothelial-derived NO can also increase the levels of IL-10 and other anti-inflammatory factors, and indirectly inhibit inflammatory cell aggregation in local inflammation, thereby reducing the inflammatory response (26). In the present study, when L-NAME, an NO synthase inhibitor, was added, the protective effect of Res was eradicated, indicating that NO exhibits a pivotal role in the protective role of Res. Similarly, when MB, a cGMP inhibitor, was added, the protective action of Res was completely inhibited, indicating that the cGMP pathway is also significant in the protective role of Res.

Previously, sirtuin 1 (SIRT1) has been shown to confer protection in various models of cardiovascular oxidative stress (27-29). SIRT1 is critical in endothelial homeostasis as it regulates endothelial NO synthase (eNOS). Endothelial-derived NO regulates blood vessel relaxation and provides atheroprotective effects. Res, a polyphenolic activator of SIRT1, has been shown to increase the expression of eNOS (30) and the combination of Res with the 3-hydroxy-3-methyl-glutaryl-CoA reductase inhibi- tors (statins) increases the activation of eNOS resulting in increased functional recovery in a model of acute myocardial infarction (31). Additionally, chronic Res treatment improved endothelium-dependent relaxation in spontaneous hypertensive rats; however, it did not increase eNOS expression (32). The role of SIRT1 in the cardioprotective process of Res remains controversial in the light of previous studies that Res is not a direct SIRT1 activator (33). Additionally, the underlying mechanisms by which Res enhances SIRT1 activity remains poorly defined and requires further studies (34).

In conclusion, the present study demonstrated that Res attenuates inflammation induced by MI/R injury. Additionally the protective effects of Res are closely associated with the inhibition of neutrophil infiltration and TNF- $\alpha$ production, the increase of NO and the cGMP signaling pathway. The present study provides insights into the mechanisms involved in the protective effect of resveratrol against myocardial ischemia/reperfusion injury and may aid the development of resveratrol as a therapy for myocardial ischemia/reperfusion injury.

\section{Acknowledgements}

This study was supported by the Health and Family Planning Commission of Jilin Province (grant no. 2012Z095).

\section{References}

1. Xiong J, Xue FS, Yuan YJ, Wang Q, Liao X and Wang WL: Cholinergic anti-inflammatory pathway: a possible approach to protect against myocardial ischemia reperfusion injury. Chin Med J (Engl) 123: 2720-2726, 2010.

2. Naidu BV, Farivar AS, Woolley SM, et al: Novel broad-spectrum chemokine inhibitor protects against lung ischemia-reperfusion injury. J Heart Lung Transplant 23: 128-134, 2004.

3. Renaud S and de Lorgeril M: Wine, alcohol, platelets, and the French paradox for coronary heart disease. Lancet 339: 1523-1526, 1992.

4. Athar M, Back JH, Tang X, et al: Resveratrol: a review of preclinical studies for human cancer prevention. Toxicol Appl Pharmacol 224: 274-283, 2007.

5. Aluyen JK, Ton QN, Tran T, et al: Resveratrol: potential as anticancer agent. J Diet Suppl 9: 45-56, 2012.

6. Piotrowska H, Myszkowski K, Zio’łkowska A, et al: Resveratrol analogue 3,4,4',5-tetramethoxystilbene inhibits growth, arrests cell cycle and induces apoptosis in ovarian SKOV-3 and A-2780 cancer cells. Toxicol Appl Pharmacol 263: 53-60, 2012.

7. Afaq F, Adhami VM and Ahmad N: Prevention of short-term ultraviolet B radiation-mediated damages by resveratrol in SKH-1 hairless mice. Toxicol Appl Pharmacol 186: 28-37, 2003.

8. Sehirli O, Sakarcan A, Velioğlu-Oğünç A, et al: Resveratrol improves ifosfamide-induced Fanconi syndrome in rats. Toxicol Appl Pharmacol 222: 33-41, 2007.

9. Xu Y, Nie L, Yin YG, et al: Resveratrol protects against hyperglycemia-induced oxidative damage to mitochondria by activating SIRT1 in rat mesangial cells. Toxicol Appl Pharmacol 259: 395-401, 2012.

10. Li F, Gong Q, Dong $\mathrm{H}$ and Shi J: Resveratrol, a neuroprotective supplement for Alzheimer's disease. Curr Pharm Des 18: 27-33, 2012.

11. López-Miranda V, Soto-Montenegro ML, Vera G, et al: Resveratrol: a neuroprotective polyphenol in the Mediterranean diet. Rev Neurol 54: 349-356, 2012 (In Spanish).

12. Lanzilli G, Cottarelli A, Nicotera G, et al: Anti-inflammatory effect of resveratrol and polydatin by in vitro IL-17 modulation. Inflammation 35: 240-248, 2012.

13. Black SC and Rodger IW: Methods for studying experimental myocardial ischemic and reperfusion injury. J Pharmacol Toxicol Methods 35: 179-190, 1996.

14. Speyer CL and Ward PA: Role of endothelial chemokines and their receptors during inflammation. J Invest Surg 24: 18-27, 2011. 
15. Khimenko PL, Bagby GJ, Fuseler J and Taylor AE: Tumor necrosis factor-alpha in ischemia and reperfusion injury in rat lungs. J Appl Physiol (1985) 85: 2005-2011, 1998.

16. Batista ML Jr, Rosa JC, Lopes RD, et al: Exercise training changes IL-10/TNF-alpha ratio in the skeletal muscle of post-MI rats. Cytokine 49: 102-108, 2010.

17. Zhu J, Liu M, Kennedy RH and Liu SJ: TNF-alpha-induced impairment of mitochondrial integrity and apoptosis mediated by caspase- 8 in adult ventricular myocytes. Cytokine 34: 96-105, 2006.

18. Meldrum DR, Cleveland JC Jr, Cain BS, Meng X and Harken AH: Increased myocardial tumor necrosis factor-alpha in a crystalloid-perfused model of cardiac ischemia-reperfusion injury. Ann Thorac Surg 65: 439-443, 1998.

19. Gurevitch J, Frolkis I, Yuhas Y, et al: Antitumor necrosis factor-alpha improves myocardial recovery after ischemia and reperfusion. J Am Coll Cardiol 30: 1554-1561, 1997.

20. Lefer AM, Ma XL, Weyrich A and Lefer DJ: Endothelial dysfunction and neutrophil adherence as critical events in the development of reperfusion injury. Agents Actions Suppl 41: 127-135, 1993.

21. Schwartz BG and Kloner RA: Coronary no reflow. J Mol Cell Cardiol 52: 873-882, 2012.

22. Ma XL, Lefer DJ, Lefer AM and Rothlein R: Coronary endothelial and cardiac protective effects of a monoclonal antibody to intercellular adhesion molecule-1 in myocardial ischemia and reperfusion. Circulation 86: 937-946, 1992

23. ChandrasekarB,Smith JB and Freeman GL: Ischemia-reperfusion of rat myocardium activates nuclear factor-kappaB and induces neutrophil infiltration via lipopolysaccharide-induced CXC chemokine. Circulation 103: 2296-2302, 2001.

24. Liu P, Hock CE, Nagele R and Wong PY: Formation of nitric oxide, superoxide, and peroxynitrite in myocardial ischemia-reperfusion injury in rats. Am J Physiol 272: H2327-H2336, 1997.
25. Li J, Wu F, Zhang H, et al: Insulin inhibits leukocyte-endothelium adherence via an Akt-NO-dependent mechanism in myocardial ischemia/reperfusion. J Mol Cell Cardiol 47: 512-519, 2009.

26. Li J, Zhang $\mathrm{H}, \mathrm{Wu} \mathrm{F}$, et al: Insulin inhibits tumor necrosis factor-alpha induction in myocardial ischemia/reperfusion: role of Akt and endothelial nitric oxide synthase phosphorylation. Crit Care Med 36: 1551-1558, 2008.

27. Alcendor RR, Gao S, Zhai P, et al: Sirt1 regulates aging and resistance to oxidative stress in the heart. Circ Res 100: 1512-1521, 2007.

28. Danz ED, Skramsted J, Henry N, Bennett JA and Keller RS: Resveratrol prevents doxorubicin cardiotoxicity through mitochondrial stabilization and the Sirt1 pathway. Free Radic Biol Med 46: 1589-1597, 2009

29. Pillai JB, Isbatan A, Imai S and Gupta MP: Poly(ADP-ribose) polymerase-1-dependent cardiac myocyte cell death during heart failure is mediated by $\mathrm{NAD}^{+}$depletion and reduced Sir2alpha deacetylase activity. J Biol Chem 280: 43121-43130, 2005.

30. Wallerath T, Li H, Godtel-Ambrust U, Schwarz PM and Forstermann U: Blend of polyphenolic compounds explains the stimulatory effect of red wine on human endothelial NO synthase. Nitric Oxide 12: 97-104, 2005.

31. Penumathsa SV, Thirunavukkarasu M, Koneru S, et al: Statin and resveratrol in combination induces cardioprotection against myocardial infarction in hypercholesterolemic rat. J Mol Cell Cardiol 42: 508-516, 2007.

32. Rush JW, Quadrilatero J, Levy AS and Ford RJ: Chronic resveratrol enhances endothelium-dependent relaxation but does not alter eNOS levels in aorta of spontaneously hypertensive rats. Exp Biol Med (Maywood) 232: 814-822, 2007.

33. Beher $\mathrm{D}, \mathrm{Wu} \mathrm{J}$, Cumine $\mathrm{S}$, et al: Resveratrol is not a direct activator of SIRT1 enzyme activity. Chem Biol Drug Des 74: 619-624, 2009.

34. Chakrabarty SP, Balaram H and Chandrasekaran S: Sirtuins: multifaceted drug targets. Curr Mol Med 11: 709-718, 2011. 